Original Research Paper

\title{
Formation Mechanism and Characterization of PANI Nanoparticles by Hybrid Chemical and Gamma Irradiation Technique
}

\author{
Shawkat Salameh Gasaymeh \\ Department of Academic Support, Abu Dhabi Polytechnic - Abu Dhabi, UAE
}

\author{
Article history \\ Received: 29-04-2018 \\ Revised: $23-08-2018$ \\ Accepted: 08-12-2018 \\ Email: sh.gasaymeh100@gmail.com
}

\begin{abstract}
Here we present additional data regarding specific doping process in nanoparticle samples of PANI. In such technique, the ionizing $\gamma-$ irradiation and (dodecylbenzenesulfonic acid), DBSA were being used as dopants and as accelerators to the oxidizing agent to polymerize aniline monomer into conductive PANI Emeraldine Salt (ES), in which PANI was produced from its monomer by this noble methodology by a single-step process. In such synthesis technique, the interactions promote a more extended conformation of PANI chains, which leads to the improvement in solubility, the thermal stability and conductivity. Hence the dielectric and optical properties of PANI nanoparticles could be improved tremendously by incorporation of a long-chain organic acid (DBSA) as the dopant ion under the influence of $\gamma$-irradiation. It was also expected that the single step, direct double doping procedure ( $\gamma$-ray and DBSA) could be used for synthesizing these nanomaterials with different long-chain dopant ions instead of the standard route of synthesizing, to avoid tedious steps involved like de-doping, re-doping etc.
\end{abstract}

Keywords: Polyaniline, PANI, Dopant, Nanocomposite, $\gamma$-Irradiation

\section{Introduction}

Polymer systems with unique properties are the recent fields of increasing scientific and technical interest, offering the opportunity to synthesize a broad variety of promising new materials, with a wide range of electrical, optical and magnetic property. Technological uses depend crucially on the reproducible control of the molecular and supramolecular architecture of the macromolecular via a simple methodology of organic synthesis. Among the conducting polymer, Polyaniline (PANI) is one such polymer whose synthesis does not require any special equipment or precautions. Conducting polymers generally show highly reversible redox behavior with a noticeable chemical memory and hence have been considered as prominent new materials for the fabrication of the devices like industrial sensors (Ebrahim et al., 2014; Khan and Mohammad, 2017; Das and Sarkar, 2016). The properties of conducting polymers depend strongly on the doping level, protonation level, ion size of dopant and water content. Conducting PANI is prepared either by electrochemical oxidative polymerization or by the chemical oxidative polymerization method (Ali et al., 2008; Tanguya et al., 2018; Hafeez et al., 2017; Ali et al., 2008; Sari and Talu, 1998; Ebrahim et al., 2007; Meftah et al., 2014; Kim et al., 2012). The emeraldine base form of PANI is an electrical insulator consisting of two amine nitrogen atoms followed by two imine nitrogen atoms. PANI (emeraldine base) can be converted into a conducting form by two different doping processes: Protonic acid doping and oxidative doping (Boeva et al., 2014; Heeger Alan, 2001; Ali et al., 2007; Bae and Jang, 2012; Mohammad et al., 2017). Protonic acid doping of emeraldine base corresponds to the protonation of the imine nitrogen atoms in which there is no electron exchange. In oxidative doping, emeraldine salt is obtained from leucoemeraldine through electron exchanges. The mechanism causing the structural changes is mainly recognized to the presence of -NH group in the polymer backbone, whose protonation and deprotonation will bring about a change in the electrical conductivity as well as in the color of the polymer (Banimahd Keivani et al., 2010; Cho et al., 2004).

The emeraldine salt of PANI is formed during protonation of the emeraldine base with organic and 
inorganic acids. As a rule, this process is referred to as doping. When PANI in the form of the emeraldine base is treated with acids, protons primarily interact with the imine atoms of nitrogen; as a result, polycations appear in this regard the acids $\mathrm{HCl}$ and $\mathrm{H}_{2} \mathrm{SO}_{4}$ are considered to be the best dopants for protonation of PANI (Ebrahim et al., 2007; Boeva et al., 2014). Because positive charges localized on neighboring nitrogen atoms increase the total energy of the polymer system, electron density tends to undergo redistribution; as a consequence, "unpairing" of the lone electron pair of nitrogen atoms occurs without any change in the amount of electrons in the system (Boeva et al., 2014; Heeger Alan, 2001; Mott and Davis, 1997). The exposure of polymers to $\gamma$-radiation induces structural defects and degradation of initial structure by scission and emission of atoms, molecules and molecular fragments (Gasaymeh et al., 2010a; 2010b; Khanna et al., 2005; Ravinder et al., 2008; Virk and Srivastava, 2001). It generates a number of charged species, ions and free radicals and induces either scission or cross-linking or both. This leads to change in density, solubility, molecular weight as well as optical and electrical properties, depends on the composition of polymer and energy of ionization radiation. However, the radiation technique has proven to be an environmentally benign and low-cost method for preparation of a large quantity of size and structure controllable metal nanoparticles (Bae and Jang, 2012; Gasaymeh et al., 2010a; Belloni, 2005; Gasaymeh et al., $2010 \mathrm{c}$ ), in addition, our obtained result is much better than those prepared with other techniques.

\section{Experimental Method}

\section{Materials}

The materials used for preparing the samples in this work dodecylbenzenesulphonic acid (DBSA, 98\%,
Aldrich) and polyvinilpirrolidone (PVP $\mathrm{Mw}=10$, Aldrich), aniline monomer (Aniline, 99.8\%, Aldrich) was distilled and stored in the refrigerator and ammonium persulfate (APS, 98\%, Aldrich) were used as received. Isopropyl alcohol was used as radical scavenger in all samples. Deionized water was prepared in the laboratory and used through this study.

\section{Procedures Setup}

PANI has been synthesized by using hybrid chemical and $\gamma$-irradiation oxidation process of aniline monomer. For this purpose, the preparation steps were done in a clean room, with appropriate amount of DBSA and PVP (as presented in Table 1 and 2 respectively) were dissolved in deionized water and maintained under vigorous stirring and heating $\left(70^{\circ} \mathrm{C}\right)$ until a homogeneous solution was obtained then cooled to room temperature and kept in a refrigerator and labeled (A). Aniline was mixed in deionized water, maintained under vigorous stirring for half an hour and then kept in a refrigerator to be used, denoted as (B). APS was dissolved in deionized water separately, maintained under vigorous stirring for half an hour and then kept in the refrigerator, denoted as (C).

It is important to note the total amount of water in $\mathrm{A}, \mathrm{B}$ and $\mathrm{C}$ was $200 \mathrm{~mL}$. All sample composition is presented in Table 2. The preparation of samples was done by adding $\mathrm{B}$ to $\mathrm{A}$ and kept under vigorous stirring for half an hour in order to get homogeneous solution and then $\mathrm{C}$ was added to them followed by the addition of Isopropyl alcohol (IPA). (The molar ratio of aniline/APS is constant for all samples). The samples were bubbled with nitrogen while the solution was under magnetic stirring for half an hour. The initially milky homogeneous solution was sealed and sent to Gamma cell expose to different doses of gamma radiation.

Table 1: The preparation steps of PANI nanoparticles

The following solutions were prepared as:

[A]: DBSA + PVP + Deionized water heated $\left(70^{\circ} \mathrm{C}\right)$ under vigorous stirring (30 $\mathrm{min}$ )

[B]: Aniline + Deionized water under vigorous stirring (30 min)

$[C]$ : APS + Deionized water under vigorous stirring $(30 \mathrm{~min})$

[D]: $5 \mathrm{~mL}$ of IPA

The quantity of PVP, IPA and water are 0.3 gram, $5 \mathrm{~mL}$ and $200 \mathrm{~mL}$ respectively in all samples. In addition, the above mixture was bubbled with Nitrogen under vigorous stirring $(30 \mathrm{~min})$ before sending in to Gamma cell

Table 2: The prepared samples of PANI nanoparticles

\begin{tabular}{lllll}
\hline Sample no. & DBSA $(\mathrm{g})$ & Aniline $(\mathrm{g})$ & APS $(\mathrm{g})$ & \\
\hline 1 & 8 & 2 & 2.9 & 10 \\
2 & 8 & 2 & 2.9 & 20 \\
3 & 8 & 2 & 2.9 & 30 \\
4 & 8 & 2 & 2.9 & \\
5 & 8 & 2 & 2.9 & 50 \\
\hline
\end{tabular}

The procedures were done as following:

$[\mathrm{A}]+[\mathrm{B}]$ under vigorous stirring $(30 \mathrm{~min})$

[C] Drop wise under vigorous stirring (30 min) [D] 


\section{Irradiation of Samples (Gamma Cell)}

All samples were irradiated with doses in range of 10 to $50-\mathrm{kGy}$ in step of $10-\mathrm{kGy}$ using Gamma cell model GC-220 $\gamma$-ray of Excel, ${ }^{60} \mathrm{Co} \gamma$-rays irradiation facility. Ionization of $\gamma$-irradiation involves the transfer of sufficient energy to a bound electron located in an atomic or molecular orbital of the irradiated material that the electron becomes free. The ejected electrons eventually lose their excess kinetic energy via electronic, vibrational and rotational excitation of the molecules in the medium. Nuclear radiation is ionizing, which on passage through matter, gives positive ions, free electrons and free radicals and excited molecules (Gasaymeh et al., 2010a). In addition, ionizing radiation also; all the valent metal can be reduced to zero (Gasaymeh et al., 2010b).

\section{A Proposed Oxidation and Reduction Processes of PANI Nanoparticles by Hybrid Chemical and Gamma Irradiation Process}

Radiation may induce modifications in polymer with the presence of DBSA. There is also the possibility of indirect formation of ionic species due to interactions between $\gamma$-ray photons and gases or polyaniline molecules (Ali et al., 2007; Banimahd Keivani et al., 2010). Hence, in addition to ionization by direct excitation with $\gamma$-rays, there is ionization caused by collisions from secondary electrons. It should also be noted that $\gamma$-ray irradiation dopes PANI and may not affect the oxidation state of the polymer (Ali et al., 2007). If this occurred, polymer degradation would take place, which is going to be discussed in FTIR analyses in the next paper. The effects from water in the irradiation-induced doping of PANI can be understood as high-energy particles passing through matter produce ionization, while a drop of water will condense faster on a charged ion than on a neutral ion as was discussed by (Compton and Allison, 1954). Therefore, the incorporation of water into the polymeric matrix could introduce some of these ionized species (or even "solvated ions" by water molecules) into the polymer backbone, thus stimulating the protonation process. It can also explain the longer times required for $\gamma$-ray doping since the absorption of water by the polymer is faster than the times involved in this doping mechanism.

However, an attempt has been made to improve the poor Process Ability of polyaniline (PANI), a major drawback in the area of conducting polymers, by using Dodecyl Benzene-Sulfonic Acid (DBSA), a bulky molecule containing a polar head and a long non-polar chain, which functions as both a surfactant and dopant at the same time. Furthermore, DBSA lower the $\mathrm{pH}$ of the medium that helps the monomer and oxidative agent to dissolve and facilities the polymerization therefore, the band gap energy will be reduced as going to be discussed in this paper. Another important reason for using DBSA is that, in an acid solution as DBSA under the influence of irradiation, the product will be in an Emeraldine Salt (ES) form of PANI, which pushes the reaction forward (Mott and Davis, 1979).

One of the possible radiolysis of water will be homo-litical breaking of the water molecule producing hydrogen that can be responsible for the polymer reduction. Since the amount of adsorbed water molecule is finite, so the effect of $\gamma$-irradiation at high dose will cause the oxidation of polymer surface (Ali et al., 2007). This result will create another type of defect called bipolaron, also with the presence of DBSA in the feed this could cause over oxidized which leads to form PANI nanoparticles in its Emeraldine Salt (ES) form, which is the conductive form of PANI. This result can be as complete evidence that $\gamma$-irradiation can be used to dope PANI (Ali et al., 2007; Banimahd Keivani et al., 2010). This is the explanation of the first proposed of $\gamma$-irradiation mechanism presented in Fig. 1A.

The second proposed of $\gamma$-irradiation mechanism presented in Fig. 1B, shows the result of the interaction of $\gamma$-irradiation with water molecules produces secondary electrons that can interact with other water molecules. This result will produce $\mathrm{OH}^{-}$anions, which proposed that $\gamma$-irradiation produces one electron from the polymeric surface creating an $\mathrm{H}^{+}$defect and the secondary electron produced interacts now with a water molecule producing an $\mathrm{OH}^{-}$anion that can be used as a counter anion to neutralize the $\mathrm{H}^{+}$defect (Ali et al., 2007; Banimahd Keivani et al., 2010; Virk and Srivastava, 2001; Pud et al., 2017). Moreover, this result also is an evidence that $\gamma$-irradiation can dope PANI and improve its properties. So that the polymer surface could undergo a slight oxidation due to contact with the environmental and according to our proposed scheme that radiolysis of water molecule can play an important role in the reduction mechanism of PANI.

However, the interaction of $\gamma$-rays with the aniline and the other continent in the feed is dominant because $\mathrm{HSO}_{3}$ is easily dissociated to $\mathrm{H}^{+}$and $\mathrm{SO}_{3}^{-}$ions by radiation. The protonation of aniline monomer by $\mathrm{SO}_{3}{ }^{-}$ produced conducting PANI nanoparticles which can be visualized by the change of color of the irradiated PVP/aniline/DBSA blend samples from colorless (milky cloddish) to dark green at 50-kGy shown in Fig. 2. Figure 2 shows the change of colour of the two samples of aniline/PVP/DBSA that irradiated with different doses. The change in colour is a big evidence of the synthesis of PANI nanoparticles. The use of this method leads to clear transparent green solution which can be directly applied on different substrates. 


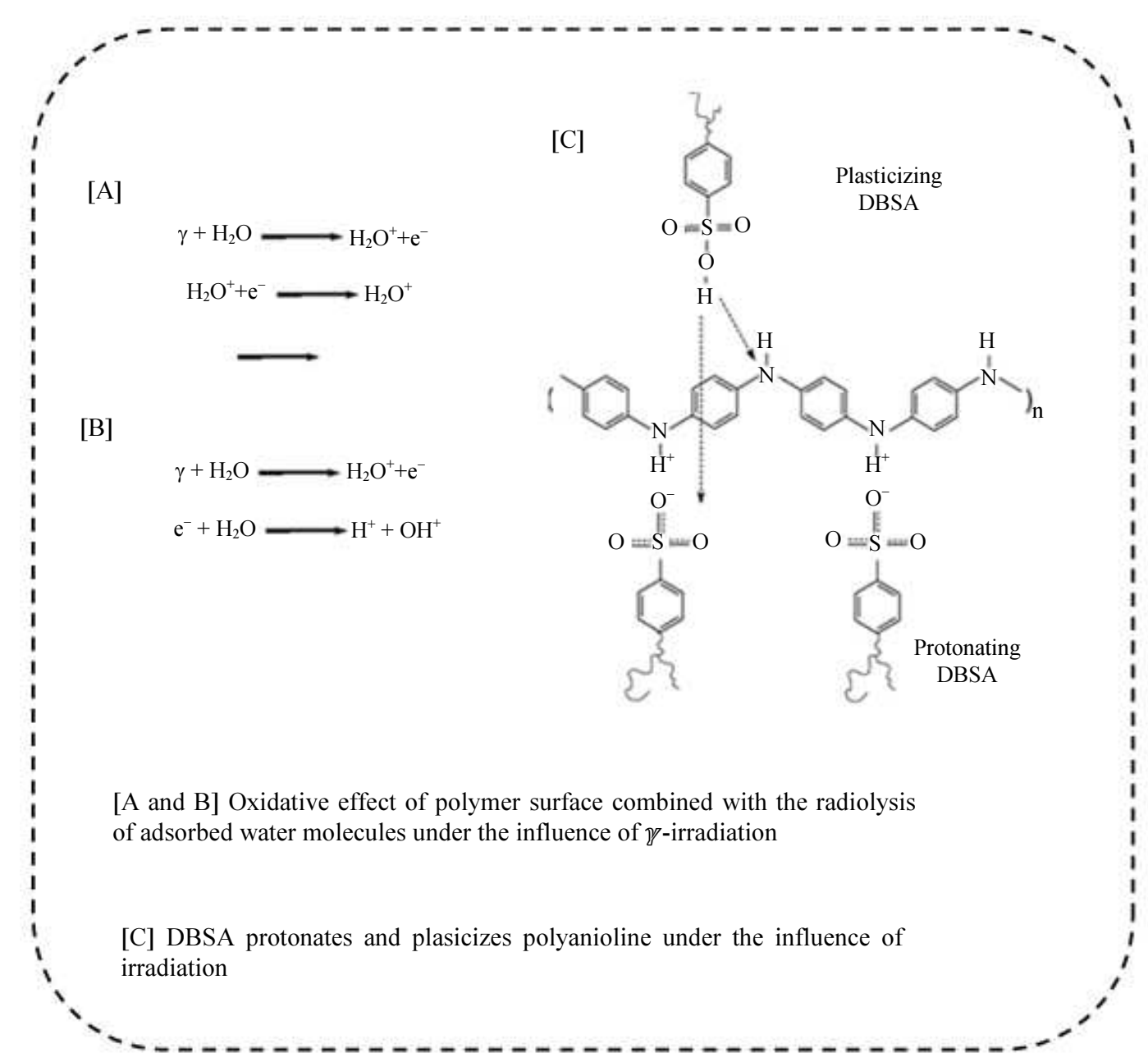

Fig. 1: A proposed oxidative effect of polymer surface combined with the radiolysis of adsorbed water molecules and DBSA protonates and plasticizes polyaniline under the influence of irradiation

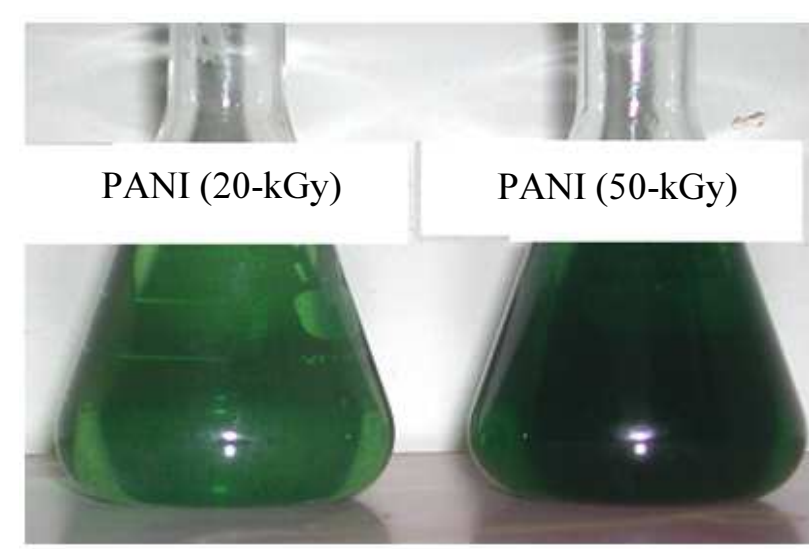

Fig. 2: The change in colour of the irradiated DBSA/PVP/Aniline composites after irradiating with 20 and $50-\mathrm{kGy}$

The charged $\left(\mathrm{SO}_{3}{ }^{-}\right)$group of PANI/DBSA has been associated with the positively charged backbone provided a solubilizing side chain compatible with organic solvents such as chloroform, $m$-cresol, dimethylsulphoxide, toluene and xylene. This technique of $\gamma$-irradiation also allows a wide range of dopants to be incorporated into the polymer to give different properties. For example, proteins can be incorporated into conducting polymers whilst retaining their biological integrity (Raymond, 2002; $\mathrm{Hu}$ and $\mathrm{Chu}, 2001)$. In addition, the solvated electrons (in the solvent water) can be easily produced during $\gamma$ irradiation, which are an important media in the system and plays a crucial role in the synthesis as proposed in Fig. 1. Employing this technique, the polymerization of monomers and the formation of inorganic nanoparticles were completed in one-step at room temperature and ambient pressure under the influence of $\gamma$-irradiation.

The possible formation mechanism of PANI Nanoparticles is shown in Fig. 3 where the anilinium cation-radicals are formed in the first step of 
polymerization. The formation of cations (positive charges) of aniline, dimmer, trimer, oligomers, etc, as well as emeraldine salt (possess positive charges in the polymer chains) is strongly correlated to anions (the negative charges). Most protonic acids provide anions (A-) in the solution. Anions tend to form ionic bonding (electrostatic force) with cations (Raymond, 2002; Hu and Chu, 2001; Shirakawa et al., 1977; Chi et al., 1992). In addition, the aniline undergoes a high degree of side chain degradation resulting mainly loss of $\mathrm{SO}_{3}^{-}$when expose to ionizing radiation. The neighboring PANI molecules thus enhancing the electrical conductivity of the composite can capture these radiolytic products (Chi et al., 1992). Ionizing irradiation of polymer causes chain scission, cross linking and change in their chemical constitution, structure and composition formation of $\mathrm{C}=\mathrm{C}$, formation of alkyne's groups, depletion of heteroatom, e.g., N, S or O (Gasaymeh et al., 2010a; 2010b; Khanna et al., 2005; Ravinder et al., 2008; Virk et al., 2001).
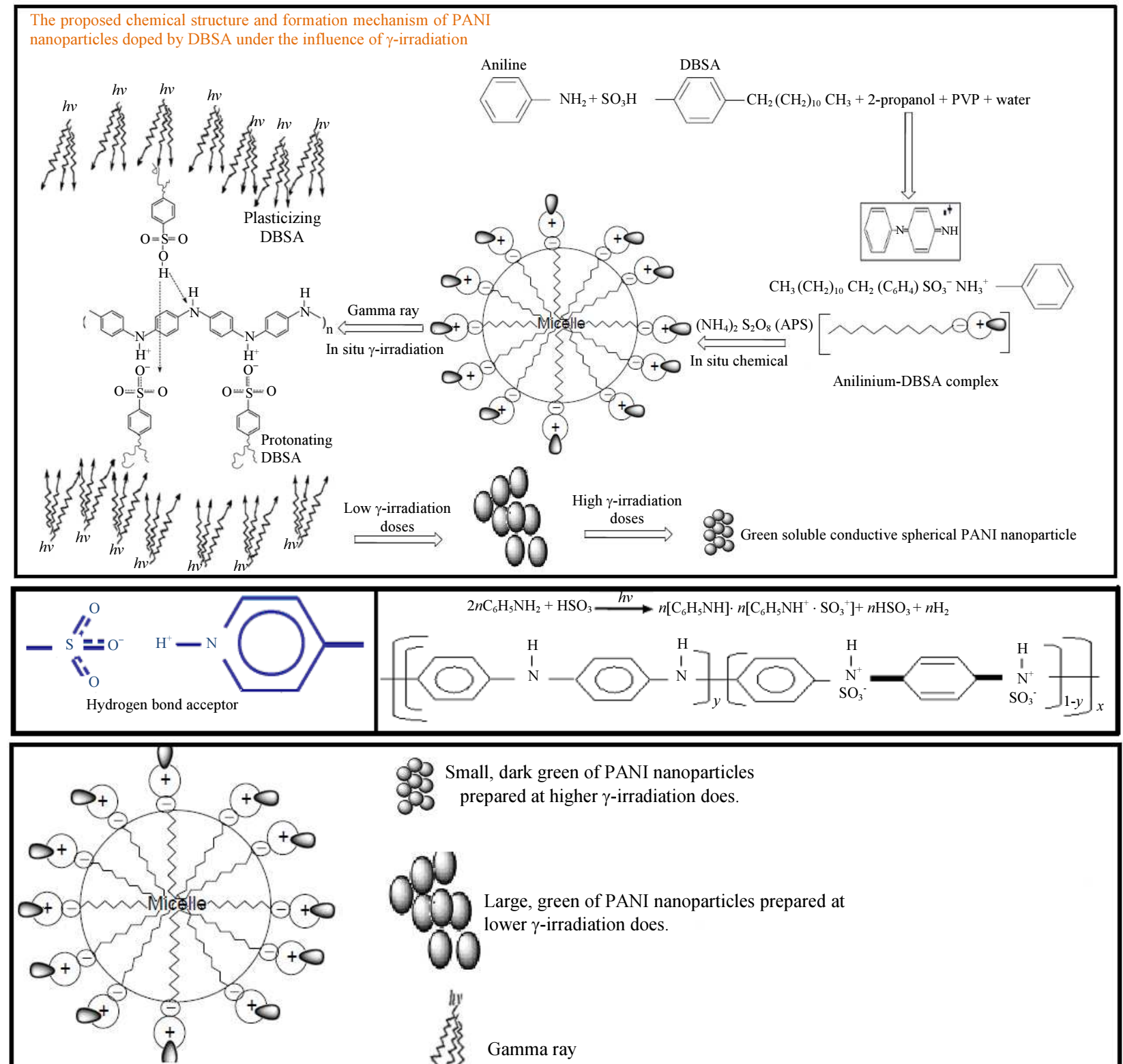

Small, dark green of PANI nanoparticles prepared at higher $\gamma$-irradiation does.

Anilinum-DBSA Micelle_complex

Gamma ray

Fig. 3: Proposed mechanism for the formation (anilinium-DBSA micelle complex) and oxidative polymerization of aniline by single step process of hybrid chemical and Gamma irradiation techniques to form spherical conductive PANI nanoparticles 
In view of many challenge, we pursue our objectives with vigilance whether the final products of conducting polymers prepared by hybrid chemical (APS and DBSA) and radiation $(\gamma)$ doping would have properties comparable with those synthesized by chemical and electrochemical methods. The chemical structure of aniline when mixed with DBSA given in the proposed scheme Fig. 3 consists of a strong benzene structure of amine group and hydrogen with $\mathrm{SO}_{3}^{-}$; this could improve the properties of PANI and modified PANI as show by our analyses. Upon exposure to $\gamma$-rays, $\mathrm{AniHSO}_{3}^{-}$undergoes high degree side chain degradation of $\mathrm{HSO}_{3}$ and these give result to mainly the loss of $\mathrm{SO}_{3}^{-}$via de-solphonation process. The radiation-induced $\mathrm{SO}_{3}{ }^{-}$acts as an oxidizing agent or oxidant that dopes the imines group of aniline and 'insitu' polymerization of imines proceeded to form soluble and electrically conductive polyaniline (PANI) in the form of Emeraldine Salt (ES) as show by our characterization, this result is similar to many published PANI (Ali et al., 2007; Mott and Davis, 1979). Generally, the conjugated PANI can exist in different chemical structures depending on the degree of oxidation or protonation. The insulating Emeraldine Base (EB) consists of equal number of oxidized (imines) and reduced (amines) units. This structure is known as leucoemeraldine salt (LB) as discussed by many authors. The conducting PANI is formed by protonation at imines $(-\mathrm{N}=)$ sites (Mott and Davis,
1979). In our case, $\mathrm{SO}_{3}^{-}$ions act as an oxidant to $\mathrm{N}^{+}$ sides and polymerized (dope) imines group into oxidized conjugated units of conducting PANI.

\section{UV-Visible Spectra pf PANI Nanoparticles}

The UV-VIS absorption spectra of the irradiated transparent green filtered PANI nanoparticles suspension in Fig. 2 obtained in our experiments reveals three prominent absorption peaks shown in Fig. 4. The first peak at around $800 \mathrm{~nm}$ assigned to the electronic transitions of $\mathrm{C}=\mathrm{N}$ bond (the shifting of electron from benzenoid ring to quinoid ring). This may be due to the creation of $\mathrm{C}=\mathrm{N}$ double bond of imines group representing the polarons in conducting PANI that gives the green color. The second peak at $423 \mathrm{~nm}\left(\pi^{*} \leftarrow \pi\right.$ transition in polaron/bipolaron) which can be assigned to the polaron band transitions and the third absorption peak at $350 \mathrm{~nm}$ corresponding to the $\pi-\pi^{*}$ transition of the benzenoid ring (Shirakawa et al., 1977), indicating that the polymer is in its Emraldine Salt (ES) state the conducting form of PANI. These three peaks constitute a typical conductive emeraldine salt spectrum of PANI. This is similar to the emeraldine salt spectrum of bulk PANI prepared by chemical experimental conditions in the solution phase (Othman et al., 2006). The longitudinal peak at $800 \mathrm{~nm}$ is characteristic of the formation of conductive polyaniline. UV-Vis spectroscopy operates on the fundamental idea of molecular orbitals.

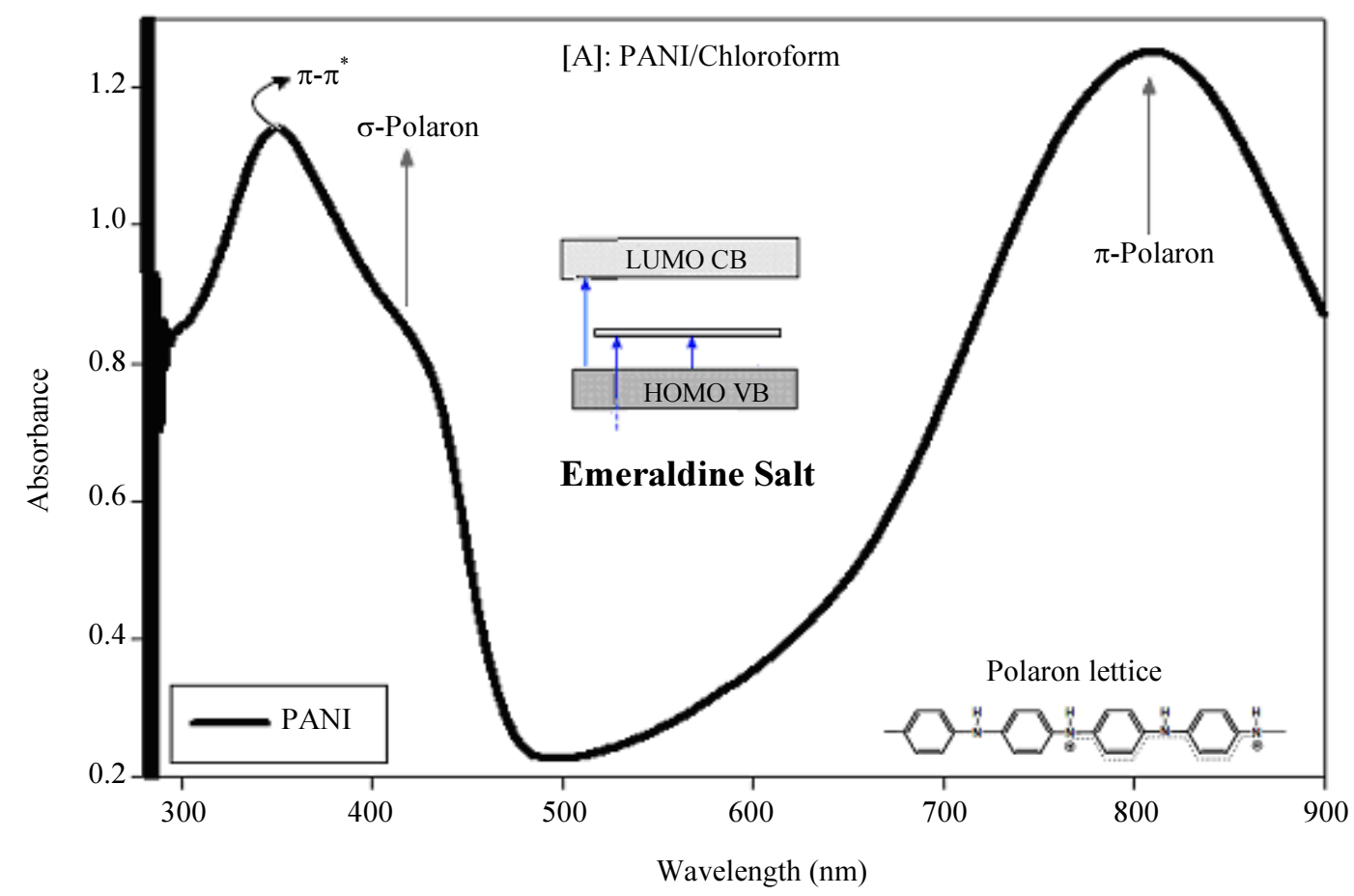

Fig. 4: UV-VIS absorption spectra of solved and filtered PANI nanoparticles in chloroform (sample 4 in Table 2) 
All molecules have molecular orbitals that are formed by adding or subtracting their corresponding atomic orbitals known as bonding and anti-bonding orbitals. The bonding orbital of a molecule is known as the Highest Occupied Molecular Orbital (HOMO) and contains the valence electrons. The anti-bonding orbital, on the other hand, is known as the Lowest Occupied Molecular Orbital (LUMO) and is normally devoid of electrons. This ideal description is valid only for single molecules. In reality, a bulk material is made up of a number of atoms brought together. In this case, the HOMOs and LUMOs of each individual atom add up to form many closely spaced orbitals, known as bands. In particular, the two discrete bands that form are known as the Conduction (CB) and Valence Bands (VB). Electrons in the valence band are tightly coupled with their respective nuclei, whereas electrons in the conduction band are somewhat separated from their respective nuclei therefore allowing for free motion within the solid.

Further, the UV-visible absorption spectra prove the proposed formation mechanism discussed above, the binding energy of $\mathrm{C}-\mathrm{C}$ bond is much stronger than $\mathrm{C}-\mathrm{H}$ and $\mathrm{C}-\mathrm{OH}$ bonds and thus, the effect of $\gamma$ - irradiation on PVP polymer at low doses is likely to cause more bond scissions of $\mathrm{C}-\mathrm{H}$ and some $\mathrm{C}-\mathrm{OH}$ side chain (Zhao et al., 2015; Shishan et al., 2003; Swallow, 1973). At higher doses, more C-OH covalent bond scissions are likely to be broken and leads to the formation of new bonds such as $\mathrm{C}=\mathrm{C}$ and $\mathrm{C}=\mathrm{O}$ of the aldehyde compounds (Cho et al., 2004). The formation of $\mathrm{C}=\mathrm{N}$ double bonds of imines group as dose increase produces green color of PANI and the intensity increases shown by the UV-vis analyses.

\section{The Optical Band Gap Energy of PANI Nanoparticles}

Generally, the optical band gap energy in a semiconductor is determined by plotting absorption coefficient $\alpha(v)$ as $(\alpha(v) h v)^{l / m}$ vs. $h v$ where $m$ represents the nature of the transition and $h v$ is the photon energy (Gasaymeh et al., 2010b). In addition, it may have different values, such as $1 / 2,2,3 / 2$ or 3 for allowed direct, allowed indirect, forbidden direct and forbidden indirect transitions respectively. However, the band gap analysis of PANI nanoparticles revealed that the band gap energy decreases DBSA concentration and/or value of gamma dose increased. The behavior observed may be due to the interference of the ionic byproducts of the oxidant, presence of free acid and dilution and more electrons are produced as gamma dose increases, which indicate more conductive emeraldine salt type with high level of protonation is produced. However, the basic principle is that photons from UV-visible light source with energies greater than the band gap energy will be absorbed by the materials under study. The absorption is associated with the electronic transitions from (HOMO) $\pi$-band to
(LUMO) $\pi^{*}$-band of electronic states (Gasaymeh et al., 2010a; 2010c; Arshak et al., 2002). The electronic transitions between the Valence Band (VB) and the Conduction band (CV) start at the absorption edge, which corresponds to the minimum energy of band gap $E_{g}$ between (LUMO) of the CB and (HOMO) of the VB.

Figure 5 shows $(\alpha(v) h v)^{2}$ Vs. $h v$ plots for the PANI nanopartices dispersed in PVP at different DBSA concentrations. The extrapolation of the curves at $(\alpha(v) h v)^{2}=0$ gives the direct band gap energy of the PANI nanoparticles in PVP based on Mott and Davis (Steckenreiter et al., 1997). However, the results show that the band gap $E_{\mathrm{g}}$ value decreases with the increase of the DBSA. Less band gap energy at higher concentration of DBSA are due to a decrease in the $\mathrm{pH}$ of the reaction medium due to higher protonation which is attributed to more conducting PANI nanoparticles formed and as more polarons in the irradiated composite reduce the band gap between VB and CB for the $\pi-\pi^{*}$ electronic transition. We found that when the DBSA was increased from 17 to $62 \mathrm{wt} \%$ the optical band gap energy decreases from 1.3 to $1.01 \mathrm{eV}$ due to higher protonation level of PANI backbone as presented in Fig. 6 .

Figure 7 shows $(\alpha(v) \mathrm{h} v)^{2}$ Vs. $h v$ plots for the PANI nanopartices dispersed in PVP prepared at different doses ranging from 10 to $50-\mathrm{kGy}$ in step of $10-\mathrm{kGy}$. The optical band gap energy decreases with the increase of the irradiation dose. The decrease in the band gap energy with increasing dose is indicated to more emeraldine nanoparticles salt is formed which leads to higher conductivity and as more polarons in the irradiated composite reduce the band gap between VB and CB for the $\pi-\pi^{*}$ electronic transition. This result is in full agreement with the proposed formation mechanism. We found that when the dose was increased from 10 to $50-\mathrm{kGy}$ the band gap decreases from 1.32 to $1(\mathrm{eV})$ illustrated in Fig. 8.

Irradiation can be used to dope polymers also a summations was made by (Ravinder et al., 2008; Virk et al., 2001; $\mathrm{Hu}$ and $\mathrm{Chu}, 2001$ ), suggested that; irradiations cause a chemical change in polymers, including cross linking, chain scission, formation of $\mathrm{C}$ $=\mathrm{C}$, formation of alkyne groups, depletion of heteroatoms, e.g., (N, S or O). In addition, years ago an extensive study by Steckenreirer and references therein (Lee et al., 2001), in polycarbonate via irradiationinduced and same result was observed.

The effect of irradiation on polymer is primarily chain scission and others, which improve the optical properties. So, by using two doping techniques gamma irradiation and DBSA acid a dramatic increase of the inter cluster interactions due to irradiation, manifested in the decrease in the band gap energy. As well as the inter-chain interactions, happens at by the same level of doping by both gamma and DBSA led them to arrive at 
a startling conclusion that the higher protonation level with more polarons is formed due to higher dopant level by using yirradiation and DBSA as discussed in the proposed formation mechanism Fig. 3. In the case of DBSA, which decreases the $\mathrm{pH}$ of the medium thereby, decreasing the band gap due to higher protonation, which causes a red shift, was also observed in our UV-vis analysis.

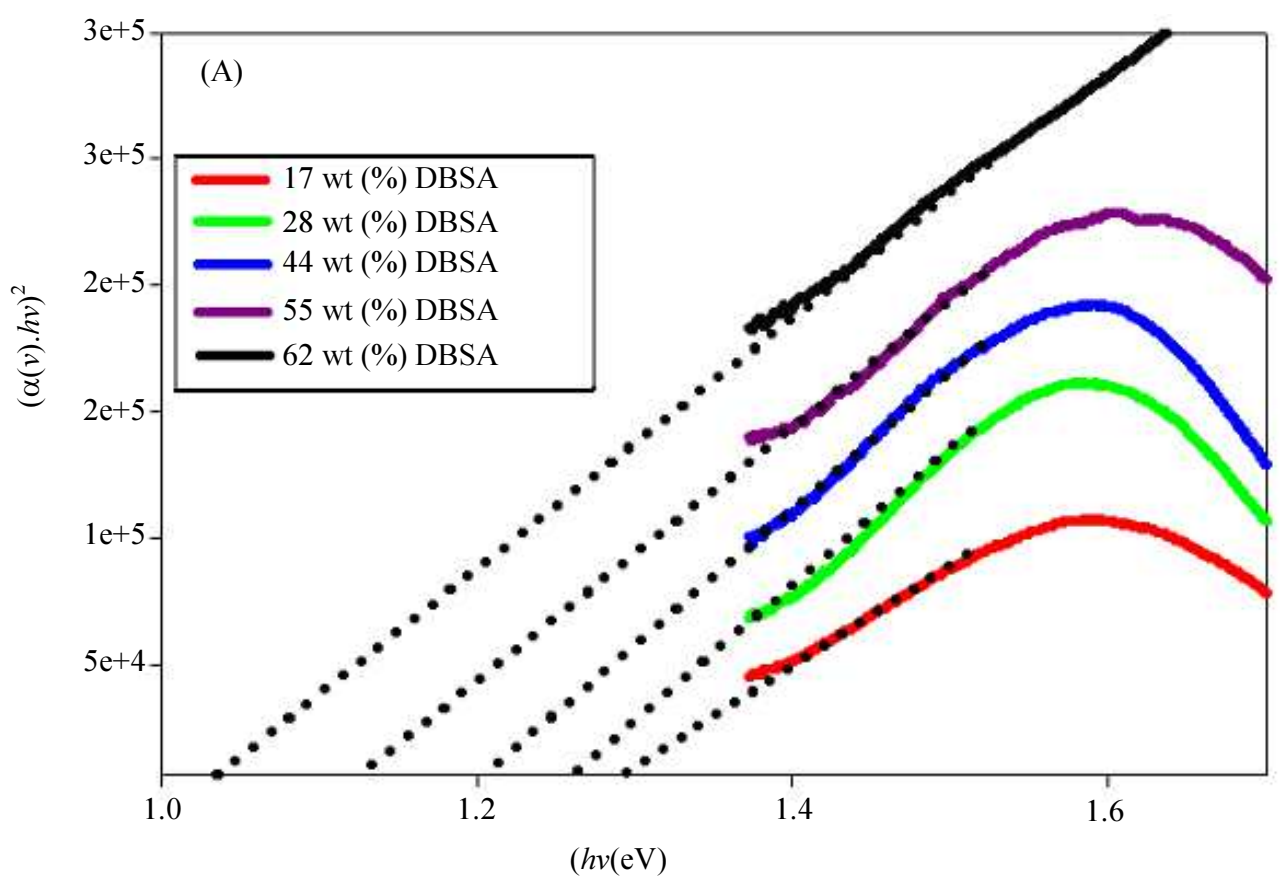

Fig. 5: The band gap of PANI nanoparticles at $16 \mathrm{wt} . \%$ of aniline, dose of $40-\mathrm{kGy}$ and different wt.\% of DBSA

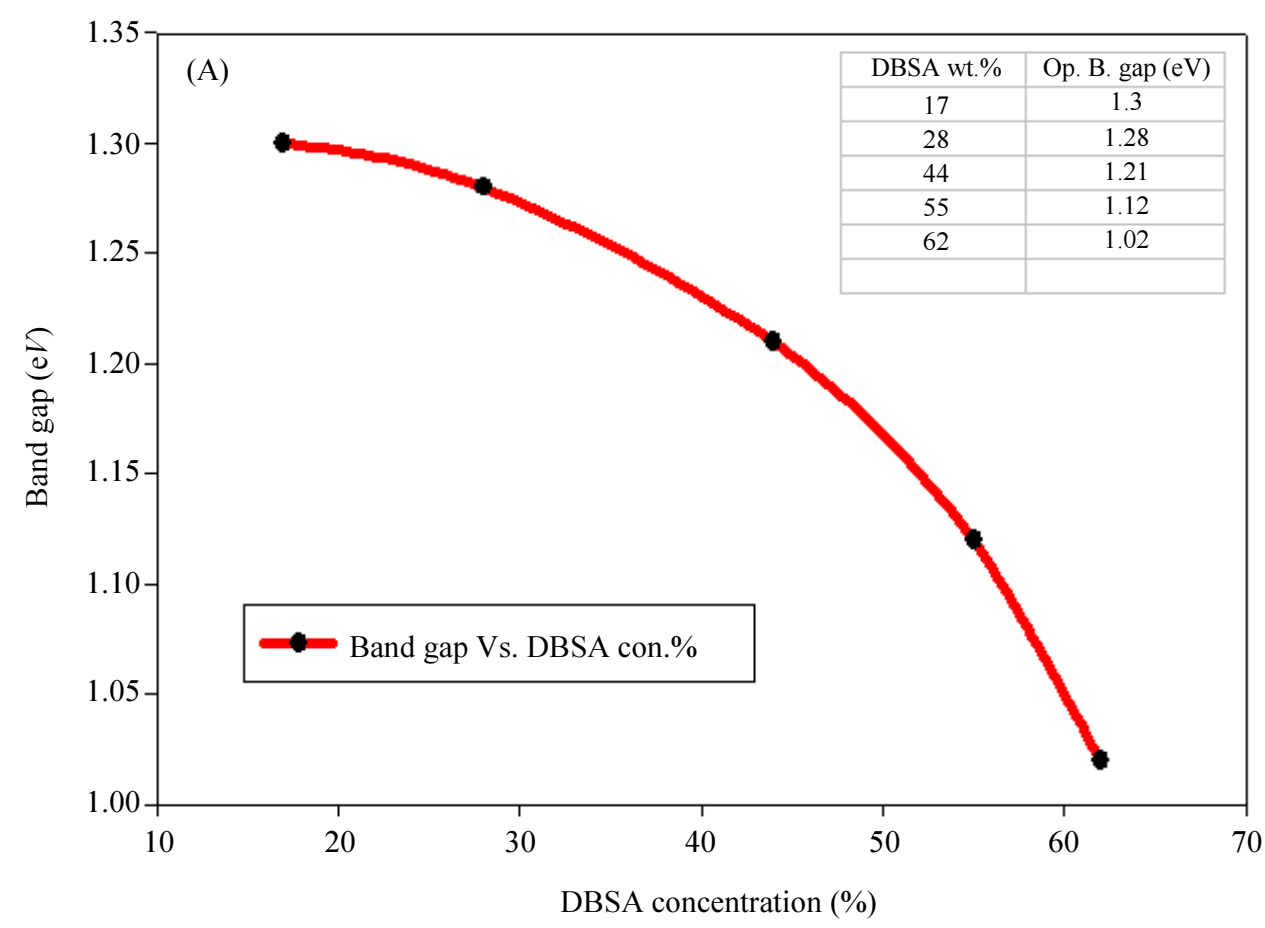

Fig. 6: A plot of band gap energy Vs. DBSA wt.\% of PANI nanoparticle synthesized at 40-kGy with 16 wt.\% of aniline 


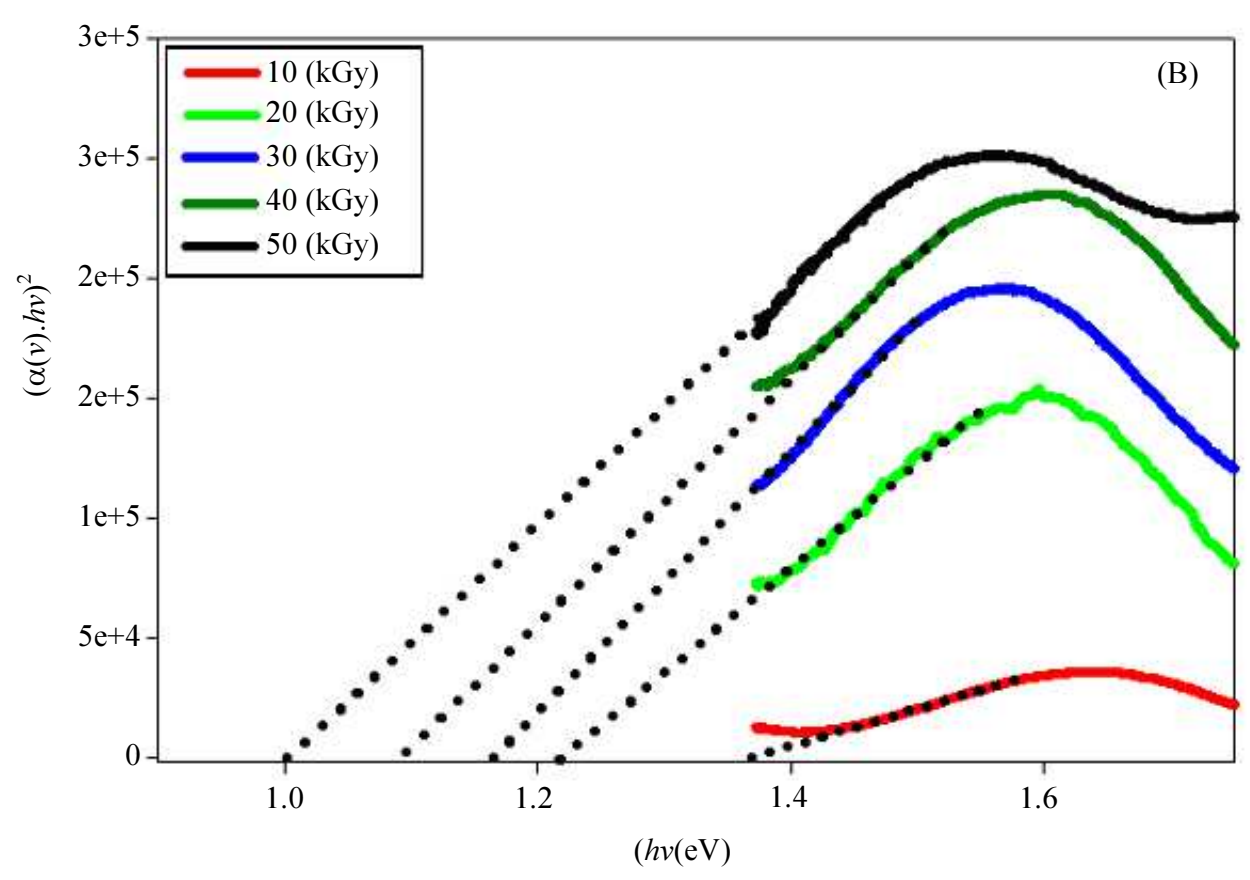

Fig. 7: The band gap of PANI nanoparticles synthesized at different doses with 55 wt. $\%$ of DBSA and 16 wt. $\%$ of aniline

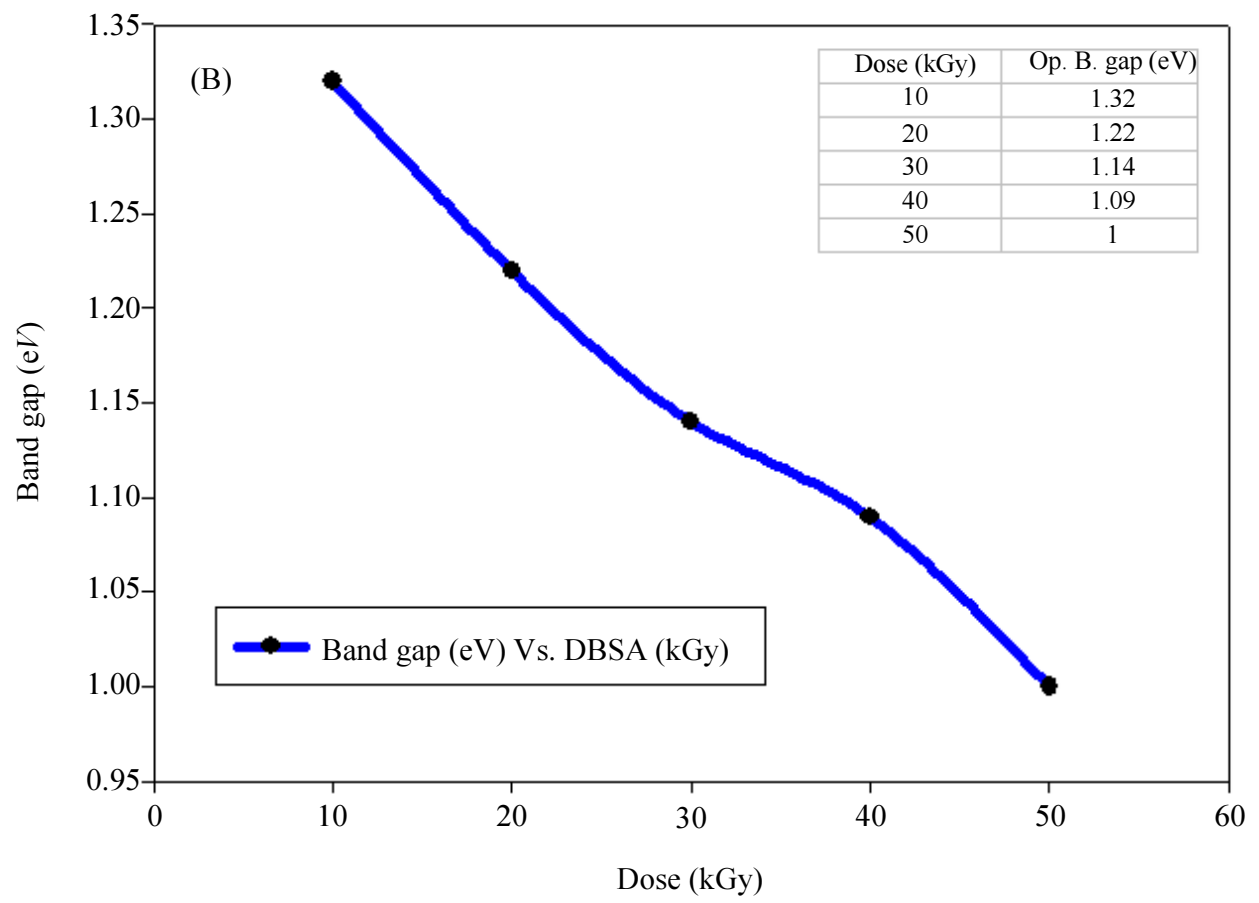

Fig. 8: A plot of the band gap of PANI nanoparticles those synthesized at different doses ranging from 10-50-kGy with 55 wt. \% of DBSA and 16 wt. $\%$ of aniline

\section{The SEM Morphology of PANI Nanoparticles}

Morphological investigations were carried out on two samples of PANI prepared at the same conditions but with different irradiation doses using Scanning Electron Microscopy (SEM) to study the influence of gamma irradiation on morphology. Both micrographs were taken at the electron operating voltage of $20 \mathrm{kV}$ and 10,000 
times magnification. The shape of PANI particle at zerokGy in Fig. 9 was grape-like and the size was estimated to be above $500 \mathrm{~nm}$. It is well known that morphology of PANI is influenced by the method of synthesis and the oxidizing agent employed. Lee, reported the shape of PANI prepared in a conventional chemical method by the SEM photograph was grape-like (Dispenza et al., 2006; Bai and Shi, 2007).

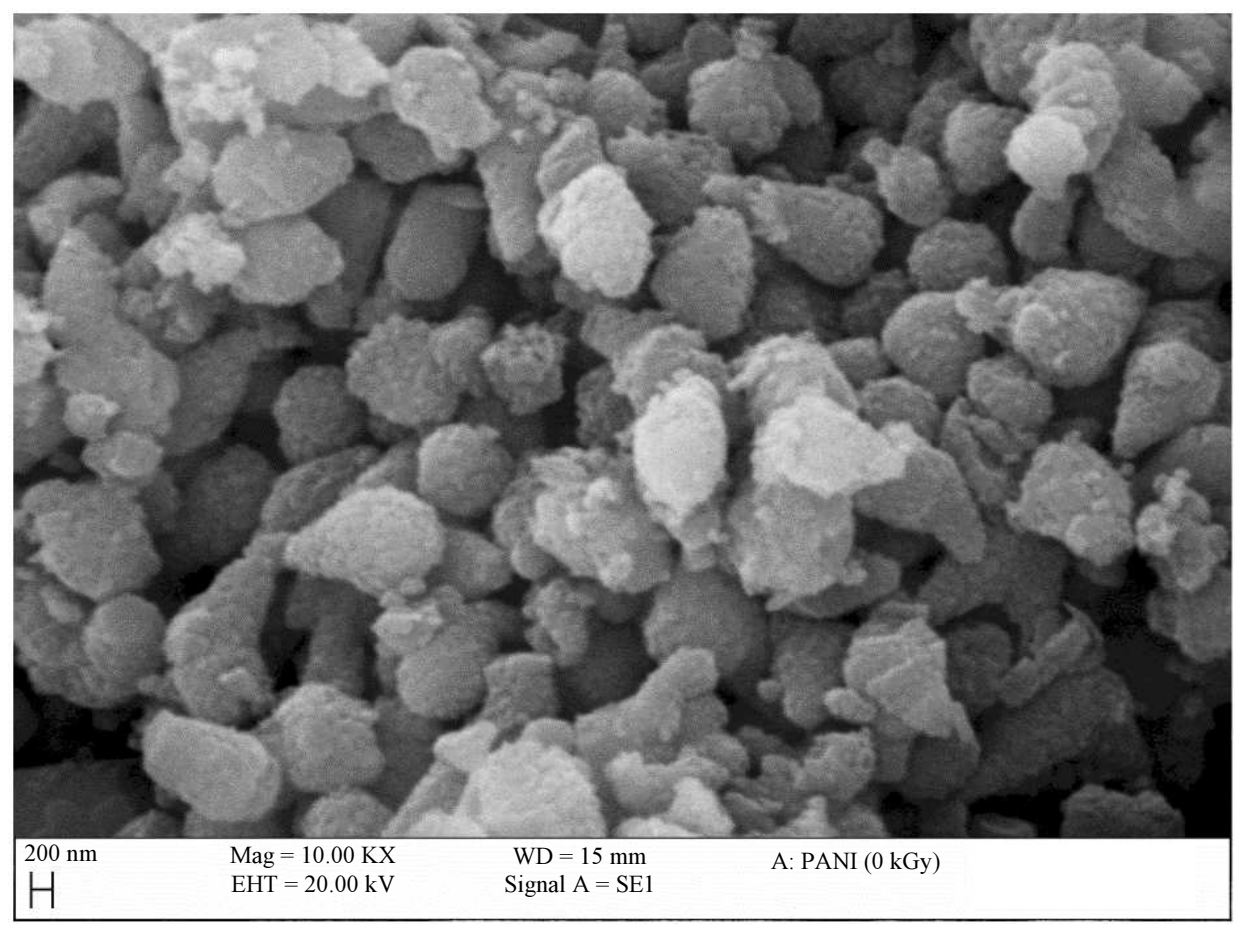

Fig. 9: SEM micrographs of PANI polymerized at 0-kGy

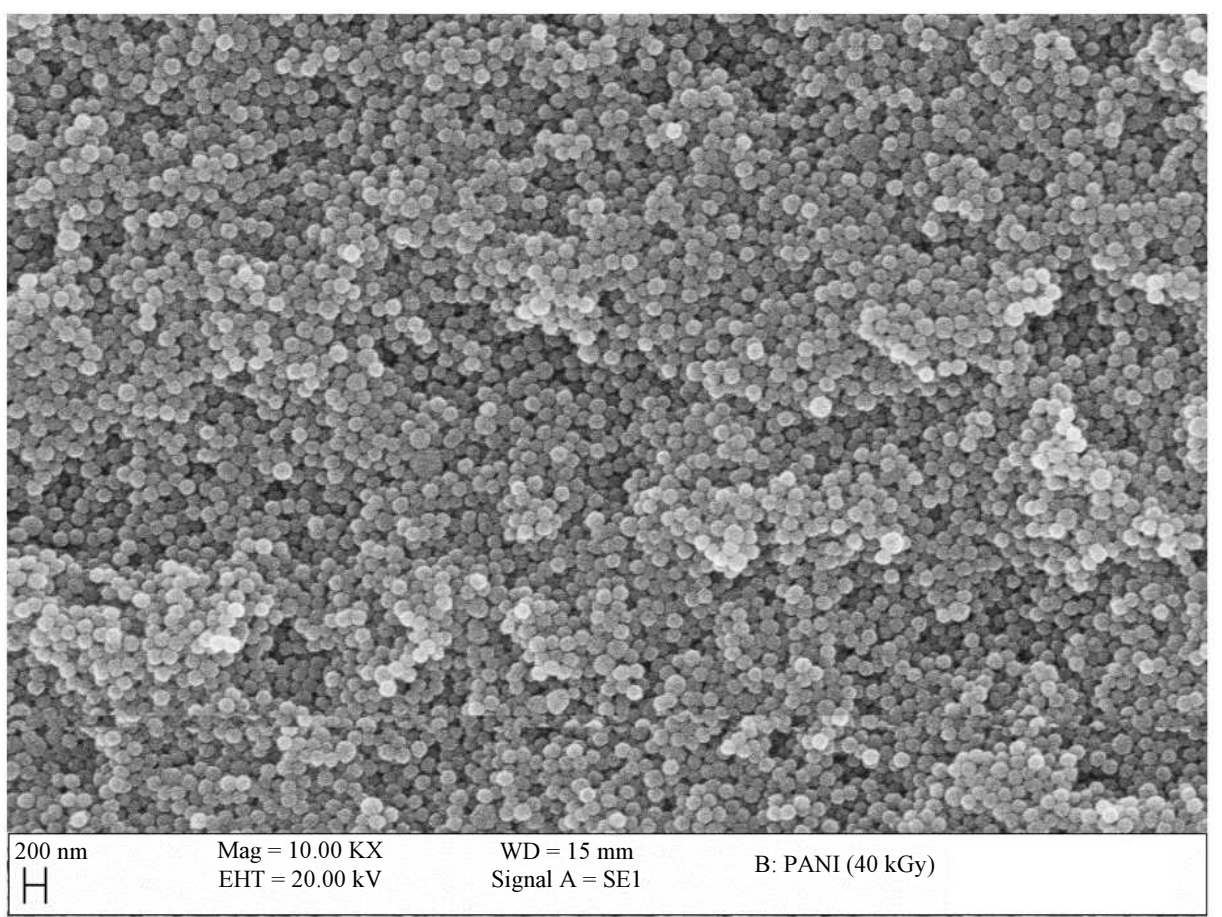

Fig. 10: SEM micrographs of PANI nanoparticles polymerized at 40-kGy 
Furthermore, Fig. 10 presented PANI nanoparticles prepared at $40-\mathrm{kGy}$, reveals the formation of conducting PANI nanostructures distributed uniformly and the diameter of spherical PANI nanoparticles was estimated to be around $100 \mathrm{~nm}$.

The SEM results show that the morphology of PANI strongly influenced by $\gamma$-irradiation and by the synthetic approach employed. The change in the morphology due to change in the $\gamma$-irradiation doses from zero to $40-\mathrm{kGy}$ may be caused by a change in conformation of the polymer. Irradiation not only can dope polymers but also it causes a number of chemical changes in polymers, including cross-linking, chain scission, formation of $\mathrm{C}=\mathrm{C}$, (Khanna et al., 2005; Ravinder et al., 2008). Moreover, there have been reported that the diameters of the PANI nanoparticles polymerized chemically with hydrochloric acid were about 100 to $150 \mathrm{~nm}$ for PVA/PANI nanocomposites (Youssef et al., 2012a) and less of that for PVP/PANI nanocomposites (Youssef et al., 2012b). This suggests that the type of binder may determine the diameter of spherical nanoparticles.

\section{Conclusion}

The purpose of this study is to establish a new method ( $\gamma$ ray radiation) synthesizing of conducting nanomaterials. We have explored how $\gamma$-irradiation techniques in the presence of protonated DBSA can syntheses and enhance the conducting and optical properties of the synthesized composites. Upon $\gamma$ irradiation DBSA undergoes high degree side chain degradation by the loss of $\mathrm{SO}_{3}^{-}$that consequently acts as an oxidant for doping the imines group of aniline and 'insitu' polymerize aniline monomer into conducting PANI in its ES state the conductive form of PANI. The formation of PANI was verified by various methods including by visual examination that the composites changed color from colorless to dark green and this was confirmed by UV-vis spectroscopy in which was used to study the optical properties of PANI nanoparticles reveals that the absorbance at $800 \mathrm{~nm}$ band for conducting PANI increased exponentially with the increase of dose $(\mathrm{kGy})$ or/and with the addition of DBSA. The higher wavelength occurs in PANI nanoparticles around the peak of $800 \mathrm{~nm}$ indicating a better protonation of the PANI backbone in our PANI nanoparticles as compared to that prepared by chemical and electrochemical methods. The doping using DBSA under the influence of irradiation has an effect on the charge carriers. On one hand, doping enhances the density and mobility of charge carrier that increases the bipolaron to polaron ratio, which leads to form conductive form of PANI.
The study of conducting polymer offers many opportunities. Even though charge transfer characteristics of conducting polymers are still not well understood, the reproducibility of electronic, optical and other properties have allowed it possible to design and fabricate conducting PANI based devices. It is proposed that the next stage of this study should include a study towards coming up with devices that would have several advantages over the conventional material made devices. Devices of particular importance would be the study on polymer-based solar cell and polymer light emitting devices. In addition, the nanostructured polymers are capable to be used in semiconductor industry, photovoltaic cell and sensor materials as well as photonic band gap materials that has not been explored thoroughly yet. Further, polymerization by $\gamma$ ray radiation should involve of several other conducting polymers.

\section{Acknowledgment}

I wish to express my deepest gratitude to Dr. Ali Al Naqbi, Director, AD Poly., and Dr.Mufeed Batarseh, Head of the Program, Academic Support Department for their constant encouragement they have renedered towards this work.

\section{Ethics}

This article is original and contains unpublished material. The corresponding author confirms that all of the other authors have read and approved the manuscript and no ethical issues involved.

\section{References}

Ali, M.A., E. Saion, Y. Noorhana, A. Kassim and K.M. Dahlan et al., 2007. Chemical modification and control of polyaniline nanocomposites conductivity by radiation technique in PVA matrix. J. Eng. Sci. Technol., 2: 280-289.

Ali, S.R., R.R. Parajuli, Y. Balogun, Y. Ma and H. He, 2008. A nnoxidative electrochemical sensor based on a self-doped polyaniline/carbon nanotube composite for sensitive and selective detection of the neurotransmitter dopamine: A review. Sensors, 8: 8423-8452. DOI: $10.3390 /$ s8128423

Arshak, A., S. Zleetni and K. Arshak, 2002. $\gamma$-radiation sensor using optical and electrical properties of manganese phthalocyanine (MnPc). Thick Film Sensors, 2: 174-184. DOI: 10.3390/s20500174

Bae, J. and J. Jang, 2012. Fabrication of carbon nanotubes from conducting polymer precursor as field emitter. J. Ind. Eng. Chem., 18: 1921-1924. DOI: $10.1016 /$ j.jiec.2012.05.004 
Bai, H. and G. Shi, 2007. Gas sensors based on conducting polymers. Sensors, 7: 267-307. DOI: $10.3390 / \mathrm{s} 7030267$

Banimahd Keivani, M., K. Zare, M. Aghaie, H. Aghaie and M. Monajjemi, 2010. Synthesis of nano conducting polymer based polyaniline and it's composite: Mechanical properties, conductivity and thermal studies. CODEN ECJHAO E-J. Chem., 7: 105-110. DOI: $10.1155 / 2010 / 963690$

Belloni, J., 2005. Nucleation, grouth and properties of nanoclusters studied by radiation chemistry: Application to catalysis. Catal. Toody, 113: 141-156. DOI: $10.1016 /$ j.cattod.2005.11.082

Boeva, Z.A. and V.G. Sergeyev, 2014. Polyaniline synthesis, properties and application. Polymer Sci., 56: 144-153. DOI: 10.1134/S1811238214010032

Chi, H.F., S.W. Lin and H.J. Huang, 1992. The mediation effect of a polyaniline coated electrode on the reduction of iron (III) ions. J. Chinese Chem. Society, 39: 213-216. DOI: 10.1002/jccs.199200036

Cho, S.M., S.Y. Park, J.Y. Hwang and H.J. Choi, 2004. Synthesis and electrical properties of polymer composites with polyaniline nanoparticles. Mater. Sci. Eng. C, 24: 15-18.

DOI: $10.1016 /$ j.msec.2003.09.003

Compton, A.H. and S.H. Allison, 1954. X-Rays in Theory and Experiment. 2nd Edn., D. Van Nostrand Company, Inc., Princeton, pp: 828.

Das, M. and D.J. Sarkar, 2016. Effect of oxidizing agent on ammonia sensing of DBSA doped polyaniline nanocomposite thin film. Mater Sci.: Mater. Electron., 27: 4109-4109.

Dispenza, C., M. Leone, C.L. Presti, F. Librizzi and G. Spadaro et al., 2006. Optical properties of biocompatible polyaniline nano-composites. J. NonCrystalline Solids, 352: 3835-3840.

DOI: $10.1016 /$ j.jnoncrysol.2006.06.017

Ebrahim, S., R. El-Raey, A. Hefnawy, H. Ibrahim and M. Soliman, 2014. A novel chloropyrifos electrochemical sensor based on polyaniline/carbon nanotubes composite. Key Eng. Mater., 605: 99-102. DOI: 10.4028/www.scientific.net/KEM.605.99

Ebrahim, S.M., A.B. Kashyout and M.M. Soliman, 2007. Electrical and structural properties of polyaniline/cellulose triacetate blend films. J. Polymer Res., 14: 423-429. DOI: 10.1007/s10965-007-9125-7

Gasaymeh, S.S., S. Radiman, L.Y. Heng, E. Saion and G.H.M. Saeed, 2010a. Synthesis and characterization of silver/polyvinilpirrolidone nanoparticles using Gamma irradiation techniques. African Phys. Rev., 4: 0006-0006.

Gasaymeh, S.S., S. Radiman, L.Y. Heng and E. Saion, 2010b. Gamma irradiation synthesis and influence the optical and thermal properties of Cadmium Sulfide (CdS)/Poly (Vinyl Pyrolidone) Nanocomposites. Am. J. Applied Sci., 7: 500-508. DOI: 10.3844/ajassp.2010.500.508
Gasaymeh, S.S., S. Radiman, L.Y. Heng, E. Saion and G.H. Mohamed Saeed, 2010c. Synthesis and characterization of silver/polyvinilpirrolidone nanoparticles using Gamma irradiation techniques. Afr. Phys. Rev., 4: 0006-0006.

Hafeez, M., M. Faheem, Z. Ul Abdin, K. Ahmad and S. Fazil et al., 2017. Synthesis and characterization of polyaniline-based conducting polymer and ITS anticorrosion application. Digest J. Nanomater. Biostruct., 12: 707-717.

Heeger Alan, J., 2001. Semi-conducting and metallic polymers: The fourth generation of polymeric materials. Angewandte Chemie Int. Edn/Rev. Modern Phys., 73: 2591-2611.

$\mathrm{Hu}$, C.C. and C.H. Chu, 2001. Electrochemical impedance characterization of polyaniline-coated graphite electrodes for electrochemical capacitorseffects of film coverage: Thickness and anions. J. Electroanalytical Chem., 503: 105-116.

DOI: 10.1016/S0022-0728(01)00385-0

Khan, M. and F. Mohammad, 2017. Thermal stability and electrical properties of DBSA doped cetyltrimethyl ammonium bromide facilitated PANI@GN nanocomposites. Int. J. Curr. Eng. Scientific Res., 4: 2393-8374.

Khanna, P.K., S. Narendra, C. Shobhit and V.A. Kasi, 2005. Synthesis of $\mathrm{Ag} /$ polyaniline nanocomposite via an in situ photo-redox mechanism. Mater. Chem. Phys., 92: 214-219.

DOI: 10.1016/j.matchemphys.2005.01.011

Kim, D.Y., J. Kim, J. Kim, A. Kim and G. Lee et al., 2012. The photovoltaic efficiencies on dye sensitized solar cells assembled with nanoporous carbon/TiO2 composites. J. Ind. Eng. Chem., 18: 15-15. DOI: 10.1016/j.jiec.2011.11.090

Lee, Y.H., C.A. Kim, W.H. Jang, H.J. Choi and M.S. Jhon, 2001. Synthesis and electrorheological characterizations of microencapsulated polyaniline particles with melamine-formaldehyde resins. Polymer, 42: 8277-8283. DOI: 10.1016/S0032-3861(01)00342-1

Meftah, A.M., E. Gharibshahi, N. Soltani, W.M.M. Yunus and E. Saion, 2014. Structural, optical and electrical properties of PVA/PANI/Nickel nanocomposites synthesized by Gamma radiolytic method. Polymers, 6: 2435-2450.

DOI: $10.3390 /$ polym6092435

Mohammad, M., S. Kamarudin, N.H. Mohamed, N. Asim and K. Sopian, 2017. Homogenization effect on nanostructure and conductivity of polyaniline nanofibre synthesis by mini-emulsion polymerization technique. IOP Conf. Series: Mater. Sci. Eng., 293: 012012-012012.

DOI: 10.1088/1757-899X/293/1/012012

Mott, N.F. and E.A. Davis, 1979. Electronic Process in Non-Crystalline Materials. 2nd Edn., Clarendon Press, Oxford. 
Othman, A., S. Elias, J.H.M.Z. Ab. Rahman, N.M. Yusof and M. Dahlan $\mathrm{Hj}$, 2006. Influence of ionizing radiation to aniline hydrochloride/PVA on conductivity. Solid State Sci. Technol., 14: 88-93.

Pud, A., O.A. Nikolayeva, L.O. Vretik, Y.V. Noskov and N.A. Ogurtsov et al., 2017. New nanocomposites of polystyrene with polyaniline doped with lauryl sulfuric acid. Nanoscale Res. Lett., 12: 493-493.

Ravinder, S., S.K. Singh, K. Ramneek and S. Lakhwant, 2008. Microstructural modifications in swift ion irradiated PET. Radiat. Phys. Chem., 77: 575-580. DOI: 10.1016/j.radphyschem.2007.06.014

Raymond, C., 2002. Chemistry/Book. 7th Edn., McGraw-Hill.

Sari, A. and M. Talu, 1998. Electrochemical polymerization and analysis of some aniline derivatives. Turk. J. Chem., 22: 301-307.

Shirakawa, H., E.J. Louis, A.G. MacDiarmid, C.K. Chiang and A.J. Heeger, 1977. Synthesis of electrically conducting organic polymers: Halogen derivatives of polyacetylene, (CH)x. J. Chem. Society Chem. Commun. DOI: $10.1039 / \mathrm{c} 39770000578$

Shishan, W., G. Ji and J. Shen, 2003. A study on ultraviolet irradiation modification of high-density polyethylene and its effect in the compatibility of HDPE/PVA fiber composites. Mater. Lett., 57: 2647-2650. DOI: 10.1016/S0167-577X(02)01344-7

Steckenreiter, T., E. Balanzat, H. Fuess and C. Trautmann, 1997. Chemical modifications of PET induced by swift heavy ions. Nuclear Instruments Meth. Physics Res. B, 131: 159-166.

DOI: $10.1016 / \mathrm{S} 0168-583 \mathrm{X}(97) 00364-9$
Swallow, A.J., 1973. Radiation chemistry: An introduction. 1st Edn., Longman, New York, ISBN-10: 058246286X, pp: 275.

Tanguya, N.R., M. Thompsonb and N. Yanc, 2018. A review on advances in application of polyaniline for ammonia detection. Sensors Actuators B, 257: 1044-1064. DOI: 10.1016/j.snb.2017.11.008

Virk, H.S. and A.K. Srivastava, 2001. Modication of optical, chemical and structural response of CR-39 polymer by $50 \mathrm{MeV}$ lithium ion irradiation. Radiat. Measur., 34: 65-67. DOI: 10.1016/S1350-4487(01)00122-6

Youssef, A.M., M.A. El-Samahy and M.H. Abdel Rehim, 2012a. Preparation of conductive paper composites based on natural cellulosic fibers for packaging applications. Carbohydrate Polymers, 89: 1027-1032. DOI: 10.1016/j.carbpol.2012.03.044

Youssef, A.M., S. Kamel, M. El-Sakhawy and M.A. El Samahy, 2012b. Structural and electrical properties of paper-polyaniline composite. Carbohydrate Polymers, 90: 1003-1007. DOI: 10.1016/j.carbpol.2012.06.034

Zhao, Y., M. Arowo, W. Wu and J. Chen, 2015. Effect of additives on the properties of polyaniline nanofibers prepared by high gravity chemical oxidative polymerization. Langmuir, 31: 5155-5163. DOI: $10.1021 /$ la504996c 